\title{
A safety study of newly generated anti-podoplanin-neutralizing antibody in cynomolgus monkey (Macaca fascicularis)
}

\author{
Takao Ukaji ${ }^{1}$, Ai Takemoto ${ }^{1}$, Ryohei Katayama ${ }^{1}$, Kengo Takeuchi2,3 and Naoya \\ Fujita $^{4}$ \\ ${ }^{1}$ Division of Experimental Chemotherapy, The Cancer Chemotherapy Center, Japanese Foundation for Cancer Research, \\ Tokyo, Japan \\ ${ }^{2}$ Division of Pathology, The Cancer Institute, Japanese Foundation for Cancer Research, Tokyo, Japan \\ ${ }^{3}$ Pathology Project for Molecular Targets, The Cancer Institute, Japanese Foundation for Cancer Research, Tokyo, Japan \\ ${ }^{4}$ The Cancer Chemotherapy Center, Japanese Foundation for Cancer Research, Tokyo, Japan \\ Correspondence to: Naoya Fujita, email: naoya.fujita@jfcr.or.jp \\ Keywords: cynomolgus monkey (Macaca fascicularis); podoplanin; platelet aggregation; tumor metastasis; toxicity \\ Received: April 06, $2018 \quad$ Accepted: August 16, $2018 \quad$ Published: September 07, 2018 \\ Copyright: Ukaji et al. This is an open-access article distributed under the terms of the Creative Commons Attribution License 3.0 \\ (CC BY 3.0), which permits unrestricted use, distribution, and reproduction in any medium, provided the original author and source \\ are credited.
}

\section{ABSTRACT}

Hematogenous metastases are enhanced by platelet aggregation induced by tumor cell-platelet interaction. Podoplanin is a key molecule to enhance the platelet aggregation and interacts with C-type lectin-like receptor 2 (CLEC-2) on platelet via PLAG domains. Our previous reports have shown that blocking podoplanin binding to platelets by neutralizing antibody specific to PLAG4 domain strongly reduces hematogenous metastasis. However, podoplanin is expressed in a variety of normal tissues such as lymphatic vessels and the question remains whether treatment of tumors with anti-podoplanin neutralizing antibodies would be toxic. Monkeys are the most suitable species for that purpose. PLAG3 and PLAG4 domains had high homology among various monkey species and human. PLAG domain deleted mutants were indicated that monkey PLAG4 domain played a more crucial role in podoplanininduced platelet aggregation than did the PLAG3 domain as in human. Moreover, newly established neutralizing antibodies (1F6, 2F7, and 3F4) targeting the monkey PLAG4 domain blocked interaction between monkey podoplanin and CLEC-2. Especially, the 2F7 neutralizing antibody strongly suppressed platelet aggregation and pulmonary metastasis. Furthermore, inhibiting podoplanin function with 2F7 neutralizing antibody exhibited no acute toxicity in cynomolgus monkeys. Our results suggested that targeting podoplanin with specific neutralizing antibodies may be an effective anticancer treatment.

\section{INTRODUCTION}

Although hematogenous metastasis of cancer cells is occurred by the migration of detached tumor cells from the primary site into the blood vessel, most cancer cells that infiltrate blood vessels are rapidly eliminated. Less than $0.1 \%$ of cancer cells survive for $24 \mathrm{~h}$ in the bloodstream [1]. Platelets are a critical component that affects tumor cell survival within the vascular system and enhances the metastasis formation [2]. Platelets facilitate the survival of circulating tumor cells by forming large tumor cell-platelet aggregates. These aggregates protect the tumor cells from immunological assault or shear stress, upregulate tumor malignancy by releasing soluble factors from activated platelets, enhance embolization in the microvasculature, and result in metastatic foci in distant organs. The importance of platelets in tumor metastasis is also supported by the 
fact that anti-platelet agents and thrombocytopenia reduce the incidence of metastasis in some experimental models [3, 4]. The administration of anti-coagulants reportedly lowers the mortality rate $[5,6]$. These findings strongly suggest that activation of platelets is intricately involved in hematogenous metastasis. Blocking platelettumor interaction might therefore make a significant contribution to reducing cancer mortality.

Podoplanin, also known as Aggrus, T1 alpha [7] and gp36 [8] is a type I transmembrane sialoglycoprotein and a key molecule in pathologic platelet aggregation and activation [9]. Podoplanin induces platelet aggregation by binding directly to C-type lectin-like receptor 2 (CLEC2) $[10,11]$. Podoplanin binding to CLEC-2 transmits platelet activation signals to the Src family kinases, Syk and phospholipase $\mathrm{C} \gamma 2[12,13]$. Podoplanin contains a characteristic conserved EDXXVTPG motif (where $\mathrm{X}$ may be any amino acid) in its extracellular domain. This motif is critical for podoplanin-mediated platelet aggregation. In a previous report, we designated this motif as the platelet aggregation-stimulating (PLAG) domain [9]. In that study, we found that podoplanin possesses three tandem repeats of the PLAG domain (PLAG1-3). However, we recently identified an additional PLAG4 domain which includes the highly conserved EDXXT motif and is closely related to the other PLAG domains [14]. Crystal structural analysis of the PLAG3 domain complexed with CLEC-2 revealed that the acidic side chains of $\mathrm{Glu}^{47}$ and $\mathrm{Asp}^{48}$ and the sialic acid attached to $\mathrm{Thr}^{52}$ on PLAG3 were recognized by basic residues on CLEC-2 [15]. We then further reported that Glu ${ }^{81}, \mathrm{Asp}^{82}$, and $\mathrm{Thr}^{85}$ residues in PLAG4 were indispensable for binding to CLEC-2 [14].

Many reports have indicated that anti-podoplanin neutralizing antibodies that block PLAG domain binding to CLEC-2 can suppress hematogenous metastasis. We generated a neutralizing antibody, MS-1, which specifically recognizes the PLAG3 domain in human podoplanin. It suppressed podoplanin-induced platelet aggregation and hematogenous metastasis in a mouse model [11]. More recently, we generated PG4D2, a neutralizing antibody recognizing the PLAG4 domain in human podoplanin and found that it strongly suppressed podoplanin-CLEC-2 binding and podoplanin-mediated hematogenous metastasis of human podoplaninexpressing tumor cells inoculated into mice [14]. On the other hand, a different reported approach has been to block podoplanin-CLEC-2 interaction by targeting the CLEC-2 receptor itself. CLEC-2 depletion by means of an anti-CLEC-2 antibody suppressed experimental hematogenous tumor metastasis and tumor growth, inhibited thrombus formation in the lungs, and improved the outcome in tumor-bearing mice [16]. Using a mouse xenograft model, Chang et al. [17] synthesized 2CP, a small compound that specifically binds to CLEC-2 and inhibits podoplanin-induced platelet aggregation in tumor cells without affecting normal hemostasis. Moreover, soluble factors such as PDGF or TGF- $\beta$ secreted from podoplanin-activated platelets have been shown to induce the growth of some tumors $[18,19]$. The blocking podoplanin-CLEC-2 interaction by neutralizing antibody contributes to suppress the tumor cell growth [11]. Based on this accumulating evidence, podoplanin appears to be a promising therapeutic target for metastatic tumors. Moreover, CLEC-2-deficient platelets have been reported to respond normally to platelet agonists, such as collagen, ADP, U46619, and PAR-4, suggesting that inhibition of podoplanin-CLEC-2 interaction may not adversely affect physiologic hemostasis [20]. Such properties are vital if this type of antibody is to be used safely as part of an anticancer therapeutic regimen.

Podoplanin is expressed in a variety of normal tissues such as lymphatic vessels, kidney podocytes, mesothelium, and alveolar epithelium. In the absence of podoplanin, the perinatal development of lung and lymphatics is lethally hindered $[21,22]$. However, it has been demonstrated in podoplanin-conditional knockout mice that there are no serious effects of podoplanin deletion after birth [23]. The question remains whether treatment of tumors with anti-podoplanin neutralizing antibodies would be toxic. Therefore, possible adverse effects and toxicities must be investigated before such antibodies can be tested clinically as antitumor agents.

The cynomolgus monkey (Macaca fascicularis) is frequently used for biopharmaceutical toxicity studies because it commonly exhibits pharmacologic responses resembling those in humans [24]. The result of nonclinical studies using monkeys is an important step before clinical testing. No toxicity studies have yet been undertaken in monkeys testing anti-podoplanin neutralizing antibodies.

This study was designed to investigate the functional PLAG domain in monkey podoplanin and the safety of anti-podoplanin neutralizing antibody administration. Experiments were performed using PLAG domain-deleted monkey mutants and with the anti-podoplanin neutralizing antibody that recognizes the monkey podoplanin PLAG4 domain.

\section{RESULTS}

\section{Involvement of the monkey podoplanin PLAG4 domain in platelet aggregation}

First, we selected podoplanin sequences of various species including closely related monkeys to human such as chimpanzee and gorilla [25] from the NCBI GenBank and compared them. A phylogenetic tree based on podoplanin protein sequences revealed that monkey podoplanin formed a monophyletic cluster with that of humans and was well-supported by high bootstrap values 
(Supplementary Figure 1A). The cynomolgus monkey podoplanin sequence was $92.6 \%$ identical to that of human podoplanin at the amino acid level. Moreover, protein sequences in the PLAG3 (47-EDDVVTPG-54) and PLAG4 (81-EDLPT-87) domains had high homology among various monkey species and completely matched human podoplanin. $\mathrm{Glu}^{47}$, $\mathrm{Asp}^{48}$, and $\mathrm{Thr}^{52}$ residues on PLAG3 and $\mathrm{Glu}^{81}, \mathrm{Asp}^{82}$, and $\mathrm{Thr}^{85}$ residues on PLAG4, critical residues for human podoplanin-CLEC-2 binding, were conserved among these species (Supplementary Figure 1B). To evaluate the contributions of both domains to CLEC-2 binding, we substituted commercially available recombinant human or mouse CLEC-2 for monkey CLEC2. The full amino acid sequence of monkey CLEC-2 was $93.4 \%$ and $64.8 \%$ identical to the human and mouse sequences, respectively. On the other hand, another group reported that four arginine residues $\left(\mathrm{Arg}^{107}, \mathrm{Arg}^{118}\right.$, $\operatorname{Arg}^{152}$ and $\operatorname{Arg}^{157}$ ) on human CLEC-2 were critical for human podoplanin binding to CLEC-2 at two positions: the interaction of the PLAG3 $\mathrm{Glu}^{47}-\mathrm{Asp}^{48}$ doublet with CLEC-2 $\mathrm{Arg}^{107}$, $\mathrm{Arg}^{152}$, and $\mathrm{Arg}^{157}$ and of PLAG3 sialic acid attached to $\mathrm{Thr}^{52}$ with CLEC-2 $\operatorname{Arg}^{118}$ [15]. In monkey CLEC-2, Lys ${ }^{118}$ was substituted for $\mathrm{Arg}^{108}$, while the $\mathrm{Arg}^{107}$, $\mathrm{Arg}^{152}$ and $\mathrm{Arg}^{157}$ residues were conserved (Supplementary Figure 1C).

We assessed the ability of the transfected $\mathrm{CHO}$ cells (CHO/mkyPDPN-WT, CHO/mkyPDPN- $\triangle$ PLAG3, $-\triangle \mathrm{PLAG} 4$, or $-\triangle \mathrm{PLAG} 3+4)$ to bind to recombinant human or mouse CLEC-2. The total podoplanin expression level in $\mathrm{CHO} / \mathrm{mkyPDPN}-\mathrm{WT}$, its deleted mutants, or $\mathrm{CHO} / \mathrm{hPDPN}-\mathrm{WT}$ was estimated using a human anti-podoplanin mAb D2-40, a potential diagnostic marker for many different types of human tumors that recognizes the PLAG1 and PLAG2 domains (33-44 aa) [26]. We selected clones possessing similar podoplanin expression signals by flow cytometric analysis (Figure 1A, left panels). The binding ability of mkyPDPN-WT to human and mouse CLEC-2 was almost same as that of hPDPN-WT (Figure 1A middle and right panels). The $\triangle$ PLAG3 mutant significantly attenuated PDPN binding to hCLEC-2 and mCLEC-2, but only showed a partial reduction of its binding capability. On the other hand, the $\triangle$ PLAG4 mutant almost completely suppressed PDPN binding to hCLEC-2 and completely abrogated to mCLEC-2, significantly. The $\triangle \mathrm{PLAG} 3+4$ mutant completely abrogated the binding capability to both CLEC-2 (Figure 1A, middle and right panels). Consistent with CLEC-2-binding activity, the $\triangle$ PLAG3 mutant had lower platelet-aggregating ability compared with WT. Both the $\triangle \mathrm{PLAG} 4$ and $\triangle \mathrm{PLAG} 3+4$ mutant had no platelet-aggregating ability (Figure 1B). These results indicate that the monkey PLAG4 domain played a more crucial role in podoplanin-induced platelet aggregation than did the PLAG3 domain.

Next, we estimated the cross-reactivity of PG4D2 with monkey podoplanin to evaluate whether acute toxicity testing with PG4D2 was feasible in monkeys. PG4D2, the neutralizing antibody specific for the human PLAG4 domain, recognized the perimeter structure from $\operatorname{Arg}^{79}$ to Leu ${ }^{83}$ (79-RIEDL-83) in human podoplanin. The homologous region in cynomolgus monkey podoplanin had $\mathrm{His}^{79}$ residue substituted for $\mathrm{Arg}^{79}$ (Supplementary Figure 1B). PG4D2 reactivity against $\mathrm{CHO} / \mathrm{mkyPDPN}$ was much lower than against CHO/hPDPN (Figure 1C, $1 \mathrm{D}$ right panels). Masking the PLAG4 domain with PG4D2 attenuated monkey podoplanin binding to human CLEC-2, but this suppressive effect was much lower than that in CHO/hPDPN (Figure 1E). PG4D2 treatment delayed the platelet aggregation induced by the interaction of $\mathrm{CHO} / \mathrm{mkyPDPN}$ with mouse platelets but with a lower suppressive effect than of human podoplanin (Figure 1F). By contrast, the MS-1 mAb reacted with both monkey and human podoplanin PLAG3 domains and suppressed monkey podoplanin-induced platelet aggregation (Supplementary Figure 2B and 2C).

\section{Establishment of antibodies recognizing the PLAG4 domain in monkey podoplanin}

To further investigate the role of the PLAG4 domain in podoplanin-induced platelet aggregation in monkeys, we established mAbs to recognize the PLAG4 domain and neutralize the interaction between monkey podoplanin and CLEC-2. A tandemly repeated cynomolgus monkey PLAG4 peptide (76-TDIHIEDLPTPEST-89) was purified as an antigen from a bacteria lysate and injected into mice. After several times of immunization, the splenocytes were collected from mice and fused with P3U1 myeloma cells (Figure 2A). Finally, three hybridoma cell lines secreting anti-PLAG4 $\mathrm{mAbs}$ were established. The three mAbs (1F6, 2F7, and 3F4) strongly recognized intact monkey podoplanin, and $2 \mathrm{~F} 7 \mathrm{mAb}$ in particular strongly recognized human podoplanin (Figure 2B).

Epitope mapping of these mAbs was established by their reactivity to recombinant monkey podoplanin lysate expressed in E. coli that harbored point mutations of each amino acid to alanine. We confirmed that these mAbs recognized the perimeter structure from $\mathrm{Asp}^{77}$ to $\mathrm{Asp}^{82}$ (1F6), from $\mathrm{His}^{79}$ to $\mathrm{Leu}^{83}(2 \mathrm{~F} 7)$, and from $\mathrm{Asp}^{77}$ to $\mathrm{Glu}^{81}$ (3F4). All these mAbs thus partly masked the PLAG4 domain (Figure 2C) and interfered with the interaction between monkey podoplanin and human CLEC-2. The 2F7 $\mathrm{mAbs}$ strongly suppressed monkey podoplanin binding to human CLEC-2 and partially suppressed the interaction of human podoplanin with human CLEC-2 (Figure 2D). Moreover, these mAbs strongly suppressed the interaction between monkey podoplanin and mouse CLEC-2, and 2F7 almost completely suppressed human podoplanin binding to mouse CLEC-2 (Supplementary Figure 3). The platelet aggregation induced by $\mathrm{CHO} / \mathrm{mkyPDPN}$ cells was strongly suppressed by $2 \mathrm{~F} 7$ and slightly so by $3 \mathrm{~F} 4$, with minimal effect by 1F6 (Figure 2E). Additionally, the 
A

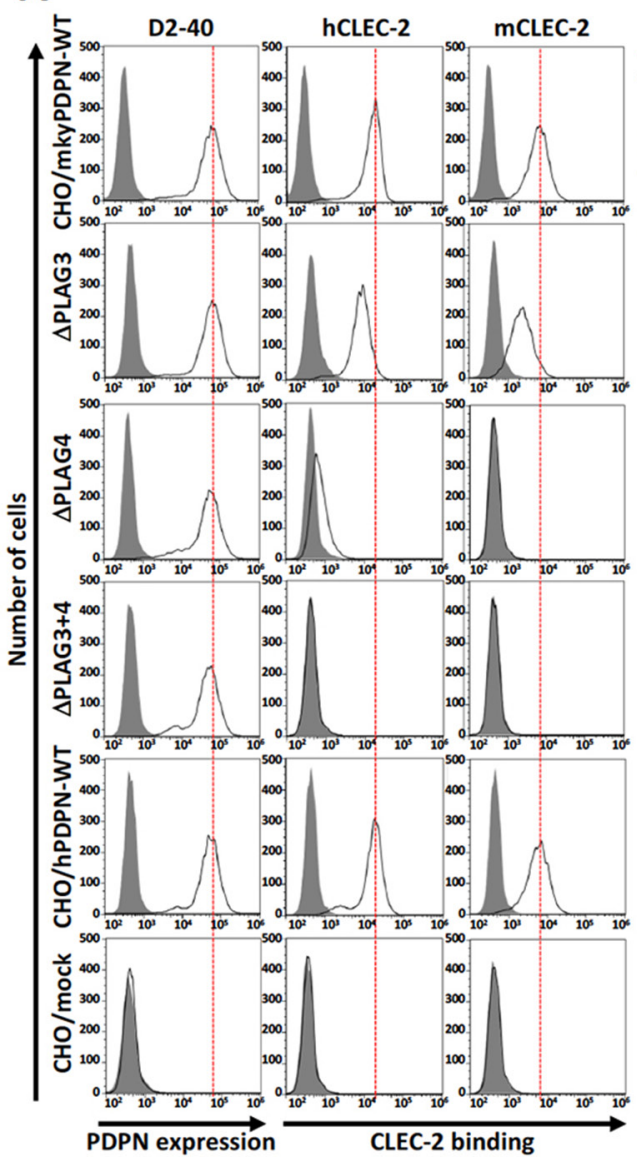

B
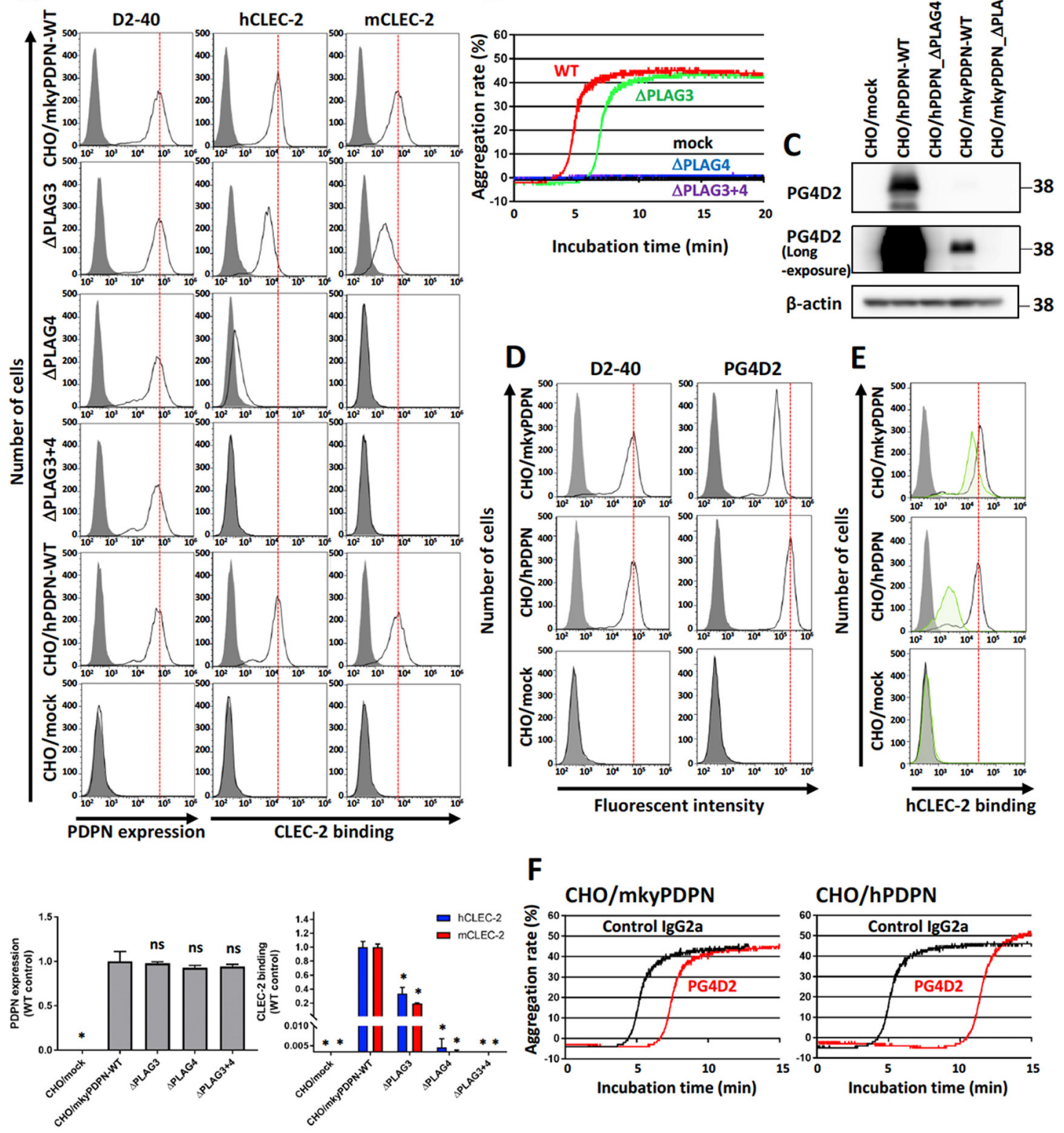

Figure 1: PLAG4 domain contributes to monkey podoplanin-induced platelet aggregation. (A) CHO cells stably transfected with an empty vector (mock), wild-type monkey podoplanin (mkyPDPN-WT), wild-type human podoplanin (hPDPN-WT), or PLAG domain-deleted podoplanin mutants (mkyPDPN- $\Delta$ PLAG3, $-\Delta$ PLAG4, and $-\triangle \mathrm{PLAG} 3+4$ ) were treated with PBS (closed areas) or antipodoplanin $\mathrm{mAb}$ (D2-40; open areas) to measure PDPN expression levels. The ability to bind with human or mouse C-type lectin-like receptor 2 (CLEC-2) was compared with PBS (closed areas), hCLEC-2-(His) ${ }_{10}(0.4 \mu \mathrm{g} / \mathrm{ml})$, or mCLEC-2-(His) $)_{10}(5 \mu \mathrm{g} / \mathrm{ml}$ ) (open areas). After washing, cells were incubated with Alexa Flour 488-conjugated second antibody. Flow cytometry data (upper) and quantitative graphs (lower) are shown. Each value in the lower graphs is the mean $\pm \mathrm{SD}(\mathrm{N}=3)$ of peak values normalized to that of CHO/PDPN-WT. ${ }^{*} P<0.05$ using Mann-Whitney $U$ test. ns, not significant. (B) Transfected $\mathrm{CHO}$ cells were incubated with mouse platelet-rich plasma (PRP). The platelet aggregation rate was estimated using an aggregometer. (C) CHO transfected with empty vector (mock), hPDPN-WT, hPDPN- $\triangle$ PLAG4, mkyPDPN, or mkyPDPN- $\triangle$ PLAG4 cells were lysed and immunoblotted with PG4D2 antibodies to PDPN or with $\beta$-actin. (D) Transfected CHO cells were treated with PBS (closed areas) or anti-PDPN antibodies D2-40 or PG4D2 (1 $\mu \mathrm{g} / \mathrm{ml})$ (open areas), and fluorescence intensity was measured. After washing, cells were incubated with Alexa Flour 488-conjugated second antibody. (E) Transfected CHO cells were incubated with $100 \mu \mathrm{g} / \mathrm{mL}$ of control IgG2a or anti-podoplanin antibody PG4D2, followed by incubation with $0.4 \mu \mathrm{g} / \mathrm{mL}$ of hCLEC-2-(His) ${ }_{10}$ protein (open areas: control IgG2a-treated samples; green areas: PG4D2-treated samples). After washing, cells were further incubated with Alexa Flour 488-conjugated anti-penta-His second antibody. CLEC-2 binding was measured by flow cytometry. Gray areas indicate the fluorescence intensity of samples not treated with CLEC-2. (F) CHO/mkyPDPN-WT or CHO/hPDPNWT cells were incubated with $10 \mu \mathrm{g} / \mathrm{mL}$ of control IgG2a or PG4D2 followed by incubation with mouse PRP. The aggregation rate was estimated using an aggregometer. 
A

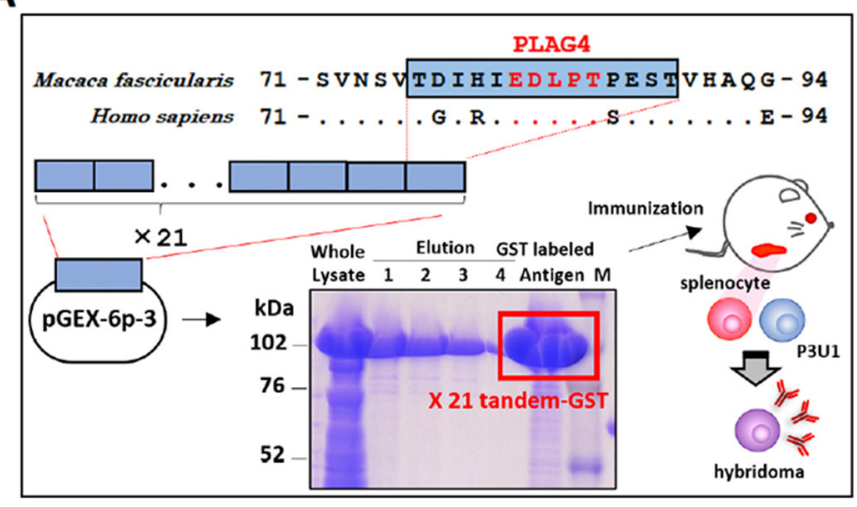

B

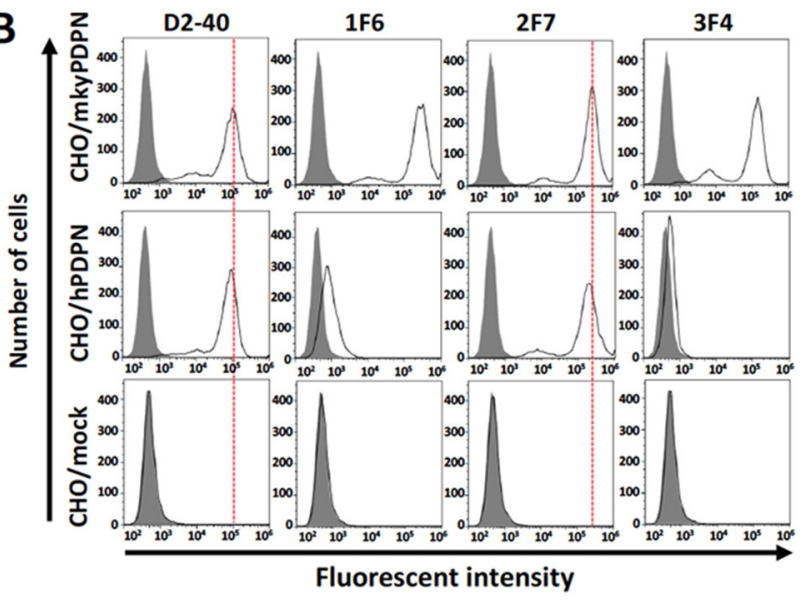

PLAG4 domain

C

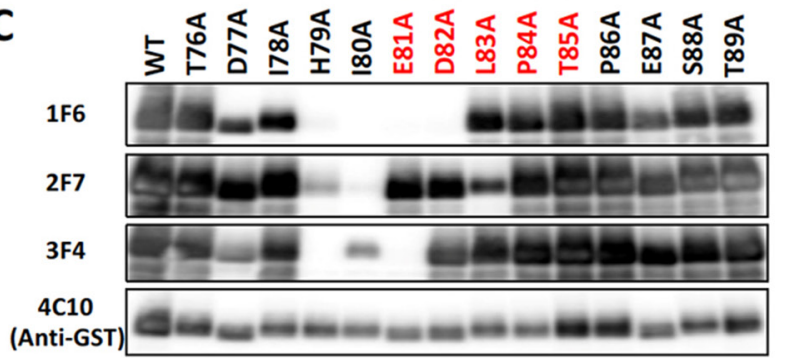

D

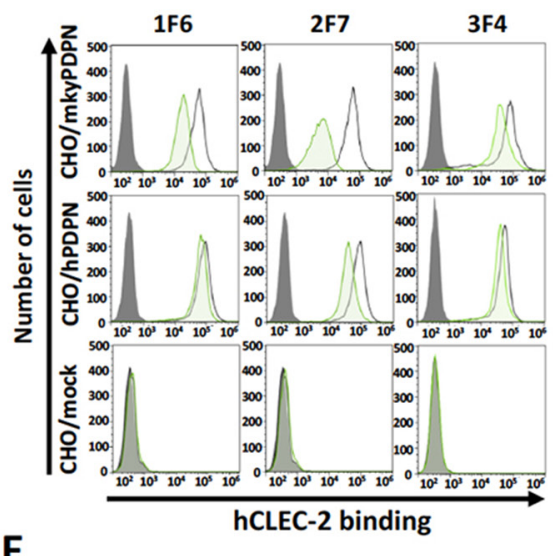

$\mathbf{E}$

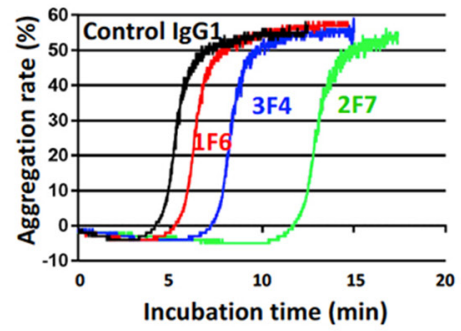

$\mathbf{F}$

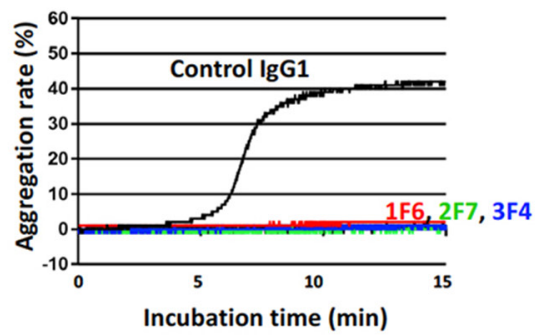

Figure 2: Establishment of monkey podoplanin-neutralizing antibodies. (A) Purification of a monkey podoplanin (mkyPDPN) immunogen to establish a hybridoma secreting anti-PDPN monoclonal antibodies (mAbs). A mkyPDPN cDNA region encoding amino acids 76-89 (226-267 bp) was tandemly connected 21 times. The cDNA fragment was inserted into a pGEX-6P-3 vector, and a glutathione S-transferase (GST)-tagged mkyPDPN peptide (76-89 aa) produced by BL21 (DE3) E. coli was purified using glutathione sepharose. $\mathrm{BALB} / \mathrm{c}$ mice were injected with the GST-tagged peptide, after which their splenocytes were fused with mouse myeloma P3U1 cells using polyethylene glycol. Hybridoma screening and antibody purification from ascites were performed. (B) CHO cells transfected with an empty vector (mock), wild-type monkey podoplanin (mkyPDPN-WT), wild-type human podoplanin (hPDPN-WT) were treated with PBS (closed areas) or antibodies (open areas), including anti-PDPN mAb D2-40, 1F6, 2F7, or 3F4 to measure PDPN expression levels. (C) GST-tagged recombinant mkyPDPN protein (WT) and its point mutants were expressed in E. coli. Cell lysates were electrophoresed and immunoblotted with antibodies to PDPN (1F6, 2F7, 3F4) or GST. The PLAG4 domain is indicated by red letters. (D) CHO/mock, CHO/ mkyPDPN-WT, or CHO/hPDPN-WT cells were incubated with $100 \mu \mathrm{g} / \mathrm{mL}$ of control IgG1 or anti-PDPN antibodies 1F6, 2F7, or 3F4, followed by incubation with $1 \mu \mathrm{g} / \mathrm{mL}$ of hCLEC-2-(His) 10 $_{0}$ (open areas: control IgG-treated samples; green areas: anti-PDPN mAb-treated samples). After washing, cells were further incubated with Alexa Flour 488-conjugated anti-penta-His second antibody. CLEC-2 binding was measured by flow cytometry. Gray areas indicate the fluorescence intensity of samples not treated with CLEC-2. (E) CHO/mkyPDPNWT cells were incubated with $10 \mu \mathrm{g} / \mathrm{mL}$ of control IgG1, 1F6, 2F7, or 3F4 mAbs followed by incubation with mouse platelet-rich plasma (PRP). The aggregation rate was estimated using an aggregometer. (F) PLAG3 domain-deleted mkyPDPN mutant cells were incubated with $10 \mu \mathrm{g} / \mathrm{mL}$ of control IgG1, $1 \mathrm{~F} 6,2 \mathrm{~F} 7$, or 3F4 mAbs, followed by incubation with mouse PRP. The aggregation rate was estimated using an aggregometer. 
platelet aggregation induced by mutated $\mathrm{CHO} / \mathrm{mkyPDPN}-$ $\triangle$ PLAG3 was completely suppressed by all three mAbs (Figure 2F).

\section{Anti-PLAG4 antibodies suppressed endogenous podoplanin-induced platelet aggregation and pulmonary metastasis}

To evaluate the role of the PLAG4 domain in endogenous podoplanin-expressing cells, we used three monkey kidney cell lines, CV-1, COS-7 and JTC-12 (Figure 3A). The mAbs 2F7 strongly suppressed platelet aggregation induced by all three lines, but $1 \mathrm{~F} 6$ did not. (Figure 3B).

To verify the role of the PLAG4 domain in monkey, we performed an in vivo metastasis assay using the $2 \mathrm{~F} 7 \mathrm{mAb}$, which had most strongly suppressed podoplanin binding to CLEC-2. Podoplanin-induced pulmonary metastasis was significantly blocked by prior administration of 2F7 mAb (Figure 3C and 3D).

\section{Acute toxicity testing of anti-podoplanin neutralizing antibody in Macaca fascicularis}

To evaluate the toxicity of anti-podoplanin antibody blocking of podoplanin-CLEC-2 binding, we prepared a large amount of purified 2F7 antibody and control IgG1 (Supplementary Figure 4A). Cynomolgus monkeys were injected with these antibodies and then evaluated for toxic effects (Supplementary Figure 4B). Hematologic and biochemical analysis of blood samples revealed no difference between those receiving the podoplanin-
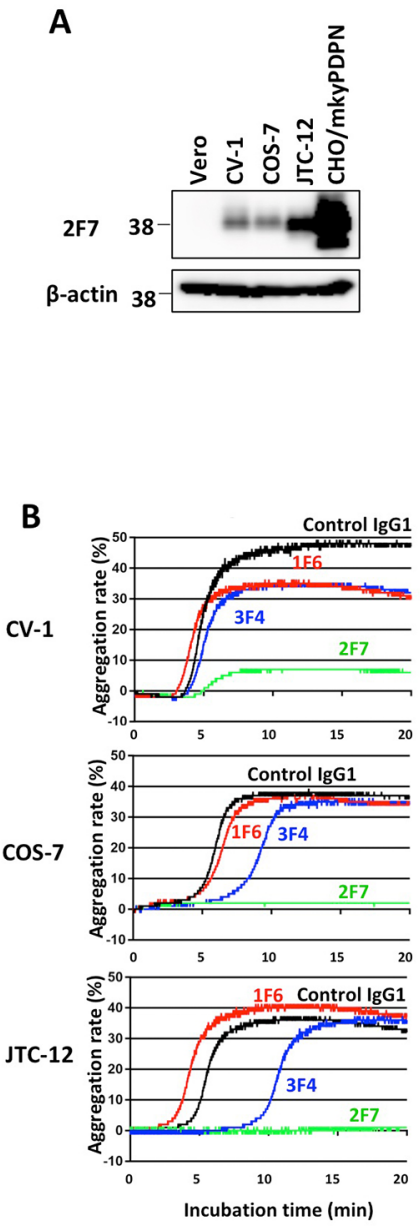

C
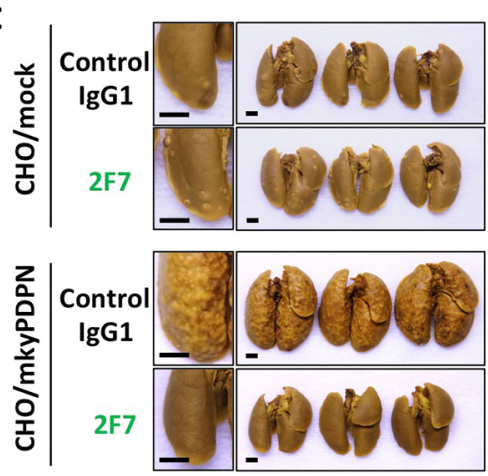

D

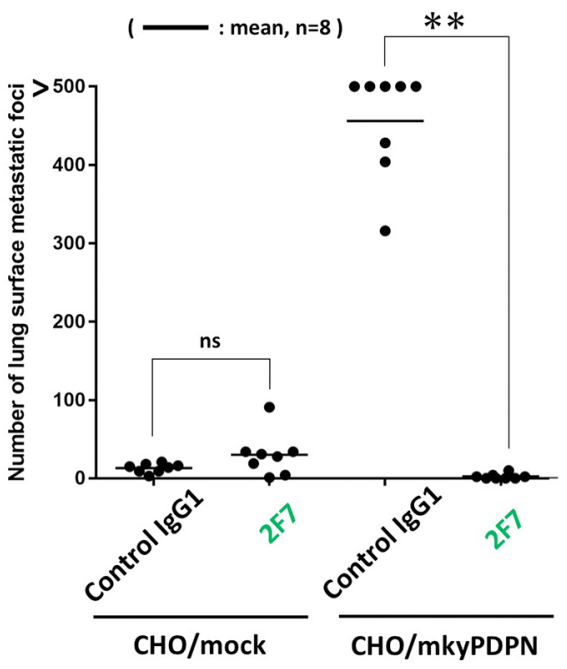

Figure 3: Inhibition of endogenous podoplanin-induced platelet aggregation and podoplanin-mediated pulmonary metastasis by anti-PLAG4 mAb. (A) CHO cells transfected with wild-type monkey podoplanin (CHO/mkyPDPN-WT) and Vero, CV-1, COS-7 or JTC-12 cells were lysed and immunoblotted with antibodies to PDPN (1F6, 2F7 or 3F4) or with $\beta$-actin. (B) CV-1, COS-7, or JTC-12 cells were incubated with $10 \mu \mathrm{g} / \mathrm{mL}$ of control IgG1, 1F6, $2 \mathrm{~F} 7$ or 3F4, followed by incubation with mouse platelet-rich plasma to assess platelet aggregation. (C) BALB/c-nu/nu mice were intravenously injected with $10 \mu \mathrm{g} / \mathrm{mouse}$ of control IgG1 or $2 \mathrm{~F} 7$. The next day, $\mathrm{CHO} /$ mock or $\mathrm{CHO} / \mathrm{mkyPDPN}-\mathrm{WT}\left(2.5 \times 10^{5}\right.$ cells/mouse $)$ were intravenously inoculated into the mice. The mice were euthanized 16 days later, and lung surface metastatic foci were counted. Representative pictures are shown. Bars, 2 mm. (D) Numbers of metastatic. Bars, mean $(\mathrm{N}=8) .{ }^{* *} P<0.01$ by a Mann-Whitney $U$ test. ns, not significant. 
neutralizing antibody and the control IgG1 monkeys (Figure 4A, and Supplementary Figure 5A and 5B). The relative weights of the brain, pituitary, thyroid, submandibular gland, heart, lung, liver, spleen, pancreas, kidney, and adrenal did not differ significantly between the two groups. However, the weights of the testis, epididymis, and prostate in the 2F7 group were higher than those of the control IgG1 group (Figure 4B). Additionally, podoplanin was strongly expressed in the monkeys' reproductive organs including the ovary, testis and epididymis (Figure 4C). Histologic evaluation with hematoxylin and eosin staining of tissue specimens from the two groups did not differ (Figure 5 , only testis, epididymis and prostate are shown). Monkeys in the podoplanin-neutralizing antibody group were mature, but the monkeys in the control IgG1 group seemed to be immature. 2F7 strongly recognized surface of cortical nodule and follicular dendritic cells of germinal center in lymph node as well as LpMab-12. On the other hand, monkey

A
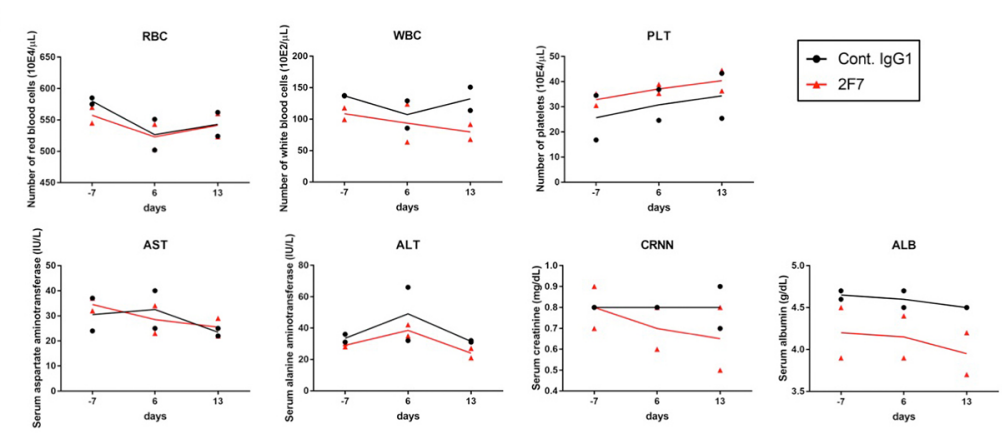

B
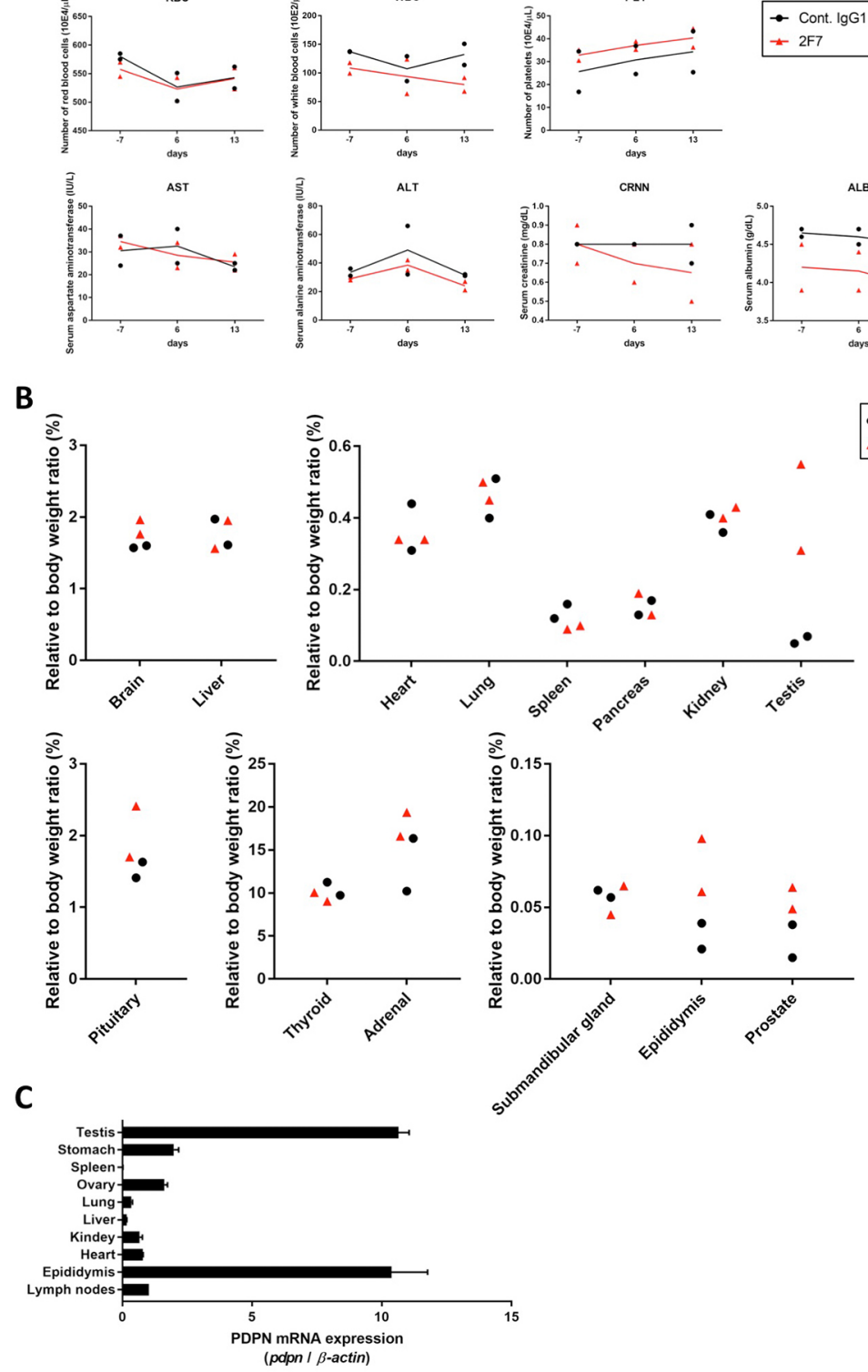
podoplanin was not immunolabeled by D2-40, FL-162 and NZ-1 (Figure 6A). Podoplanin expression was detected by 2F7 in convoluted seminiferous tubules of testis (Figure $6 \mathrm{Ba}$ and $6 \mathrm{Bb}$ ), ducts of epididymis (Figure $6 \mathrm{Bc}$ and $6 \mathrm{Bd}$ ), and interstitial connective tissue of prostate (Figure $6 \mathrm{Be}$ and 6Bf), but not by control IgG1 (Supplementary Figure 6).

\section{DISCUSSION}

Podoplanin overexpression has been detected in wide range of tumor cells, including squamous cell carcinoma [27], mesothelioma [28], glioblastoma [29], bladder cancer [30], angiosarcoma [31], brain tumors
[32], testicular seminoma [33], and osteosarcoma [34]. It is well recognized that podoplanin expression is correlated with a poor prognosis and tumor malignancy in lung carcinomas, oral squamous cell carcinomas, and breast cancers [35-39]. Interference with podoplanin-induced platelet aggregation and activation is directly associated with inhibition of podoplanin-mediated tumor metastasis [40]. As described above, a neutralizing antibody targeting the podoplanin PLAG domain is a strong candidate to target podoplanin-induced hematogenous metastasis. Because our previously established neutralizing antibodies suppress interaction between podoplanin and CLEC-2, they are capable of inhibiting hematogenous metastasis
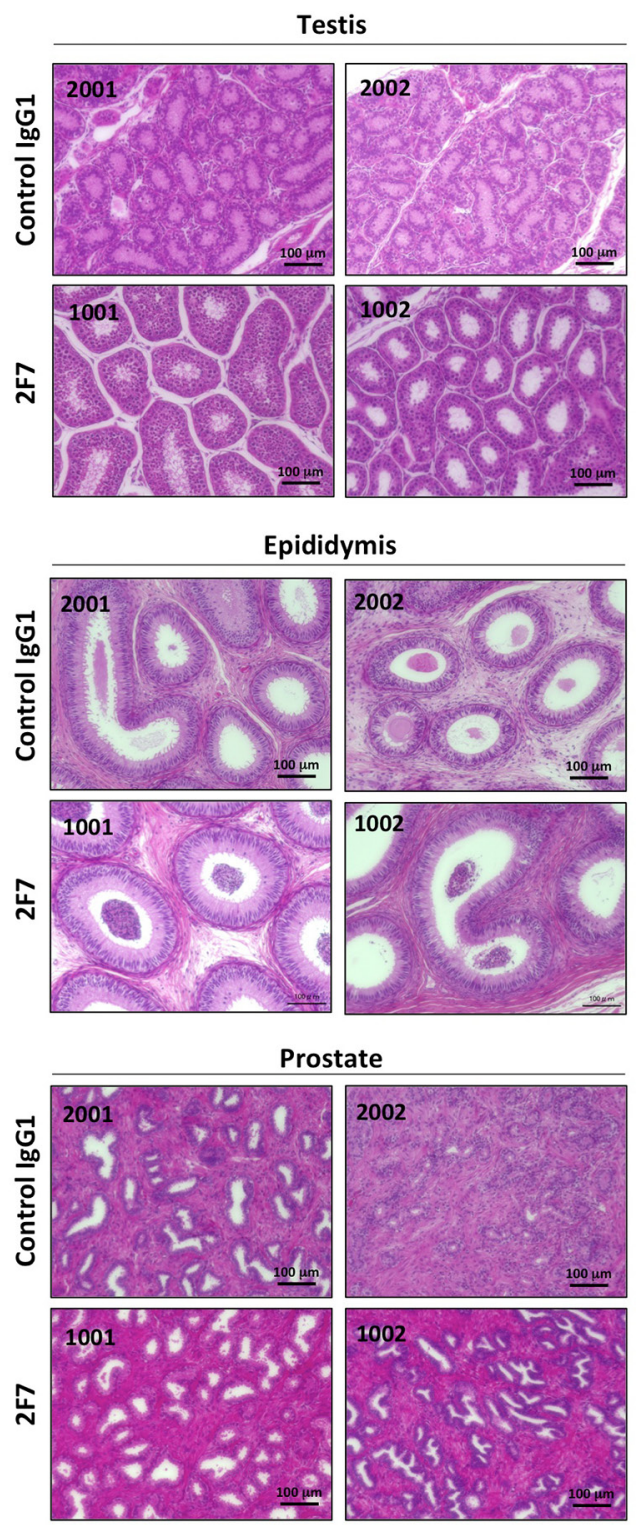

Figure 5: Histopathological examination after injection of anti-podoplanin neutralizing antibodies in Macaca fascicularis. Representative images of sections of the testis, epididymis, and prostate tissue from monkeys injected with control IgG1 (identity number: 2001 and 2002) or anti-podoplanin antibody 2F7 (identity number: 1001 and 1002). Each section was treated with hematoxylin-eosin. 
of podoplanin-expressing cancer cells $[11,14]$. Although there have been many reports of podoplanin-neutralizing antibodies capable of inhibiting podoplanin-CLEC-2 binding, the toxicity associated with such antibodies has not been investigated.

To identify PLAG domains critical for CLEC-2 binding in monkeys. We used human and mouse CLEC-2 to evaluate the interaction between monkey podoplanin and CLEC-2. Both human and monkey CLEC-2 crossreacted with mkyPDPN-WT at the same signal intensity as with hPDPN-WT (Figure 1A middle and right panel). The reason for the cross-reactivity with monkey podoplanin is likely the fact that the critical residues $\left(\mathrm{Glu}^{47}, \mathrm{Asp}^{48}\right.$, and $\mathrm{Thr}^{52}$ residues on PLAG3 and Glu ${ }^{81}, \mathrm{Asp}^{82}$, and $\mathrm{Thr}^{85}$ residues on PLAG4) on human podoplanin that bind human CLEC-2 were completely conserved on monkey podoplanin (Supplementary Figure 1B). Moreover, 3 of 4
Arg residues on CLEC-2 critical for podoplanin binding in humans were also conserved on monkey CLEC-2 (Supplementary Figure 1C). Mouse CLEC-2 also crossreacted with monkey podoplanin. In previous research, we substituted mouse platelets for human podoplanininduced platelet aggregation assays because mouse CLEC-2 cross reacts with human podoplanin. Because monkey podoplanin amino acid sequences were $92.6 \%$ homologous with human podoplanin, we speculated that monkey podoplanin might also bind mouse CLEC-2. We therefore demonstrated that either human or mouse CLEC2 could be substituted for monkey CLEC-2 to evaluate the interaction between monkey podoplanin and CLEC-2.

Deletion of the PLAG3 or PLAG4 domains significantly attenuated CLEC-2 binding and monkey podoplanin-induced platelet aggregation, an effect especially obvious with DPLAG4 mutants, with almost

A
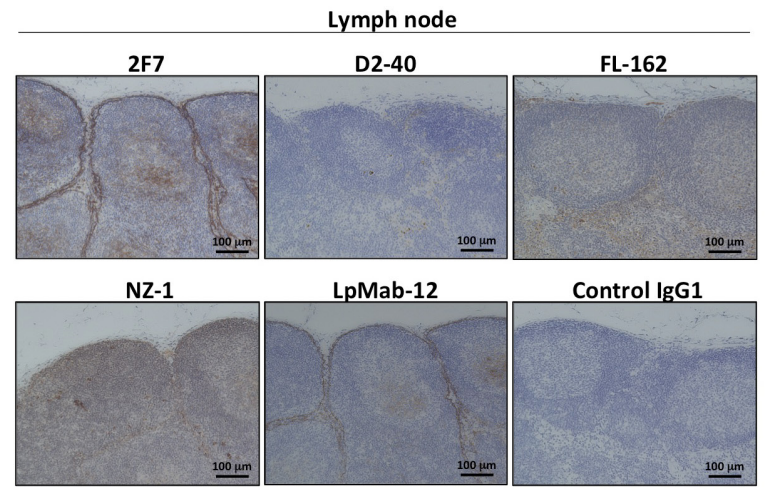

B
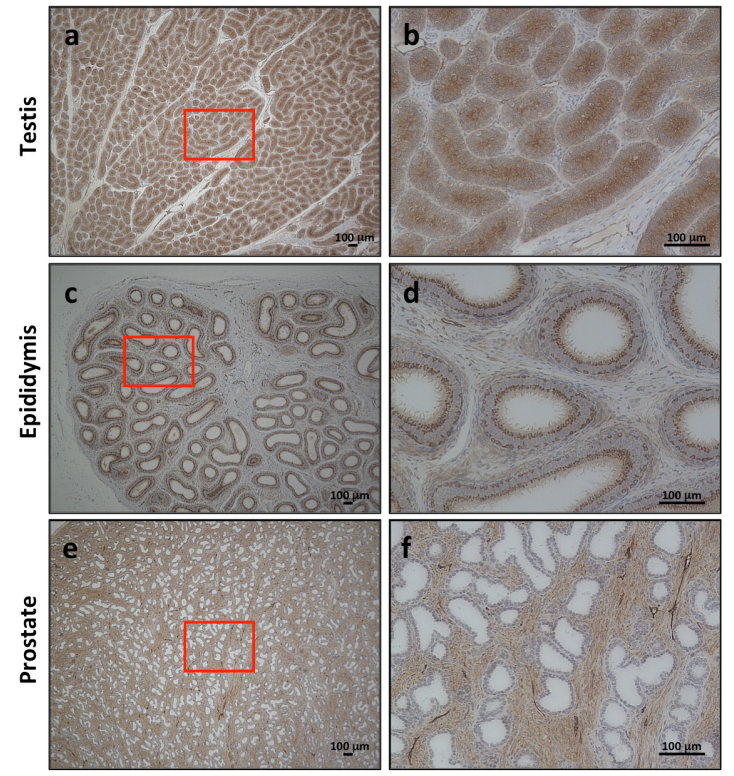

Figure 6: Podoplanin expression in normal tissue of Macaca fascicularis by immunohistochemical staining. (A) Detection methods of podoplanin expression in lymph node were evaluated by anti-podoplanin antibodies: 2F7, D2-40, FL-162, NZ-1, LpMab-12, or control IgG1, respectively. Only 2F7 and LpMab-12 were recognized the podoplanin-expressing cells. Representative images of immunohistochemical staining were shown. (B) Testis (a and b), epididymis (c and d), or prostate (e and f) in Macaca fascicularis were immunolabeled with 2F7 for localization of podoplanin and representative images were shown. The higher magnification of area surrounded by red line ( $\mathrm{a}, \mathrm{c}$ or e) was shown in b, d, or f, respectively. 
complete loss of CLEC-2 binding and platelet aggregation (Figure 1A and 1B). Therefore, we demonstrated that the PLAG3 and PLAG4 domains in the monkey, and particularly PLAG4, play a crucial role in podoplanininduced platelet aggregation as they do in humans. The PG4D2 neutralizing antibody specific to the human PLAG4 domain was not suitable for assessing the toxicity of podoplanin inhibition in monkeys because it had less affinity for monkey podoplanin and barely suppressed monkey podoplanin-induced platelet aggregation (Figure $1 \mathrm{D}$ and $1 \mathrm{~F})$. On the other hand, our newly established neutralizing antibodies (1F6, 2F7, and 3F4) targeting the monkey PLAG4 domain blocked interaction between monkey podoplanin and CLEC-2. The 2F7 neutralizing antibody could inhibit platelet aggregation induced by $\mathrm{CHO}$ cells expressing monkey podoplanin and by monkey podoplanin endogenously expressing cell lines (Figure $2 \mathrm{E}$ and $3 \mathrm{~B}$ ). It also strongly suppressed the pulmonary metastasis of $\mathrm{CHO}$ cells expressing monkey podoplanin (Figure 3C and 3D). In our previous results obtained by using several anti-podoplanin neutralizing antibodies, pulmonary metastasis of cancer cells was suppressed by the intravenous injection of anti-podoplanin neutralizing antibody on 1 hour to 3 days before tumor injection. On the other hand, the suppressive effects of antibodies became less remarkable when the administration was on 1 to 7 days after injection. We suggest that administration on 1 to 3 days before cell injection will maximize their metastasisinhibitory effects. Each neutralizing antibody partially masked the PLAG4 domain, but 2F7 most strongly suppressed its binding to CLEC-2. 2F7 recognizes the same region as the PG4D2 epitope, i.e., from $\mathrm{His}^{79}$ to $\mathrm{Leu}^{83}$ (79-HIEDL-83) of monkey podoplanin in 2F7 and $\mathrm{Arg}^{79}$ to $\mathrm{Leu}^{83}$ (79-RIEDL-83) of human podoplanin in PG4D2 (Figure 2C). Therefore, masking the perimeter structure on podoplanin from 79 to 83 aa, which included the PLAG4 domain, appears to induce strong suppression of binding. These findings indicate that interaction between monkey podoplanin and CLEC-2 can be suppressed by PLAG4 neutralizing antibodies. The PLAG4 domain in the monkey has as crucial a role in podoplanin-mediated hematogenous metastasis as in humans.

Having demonstrated the efficacy of the antibodies we developed to neutralize podoplanin function, we conducted acute toxicity testing in cynomolgus monkeys given excess amounts of the podoplanin-neutralizing antibodies. As noted above, podoplanin expression is reportedly expressed on lymphatic endothelial cells [31], kidney podocytes [41, 42], mesothelium, and alveolar epithelium. Podoplanin helps to separate the blood and lymphatic vascular systems and regulates the development of lymphatic vessels [13, 22, 43]. Podoplanin acts to regulate the initial stage of osteocyte differentiation [23]. It serves as a marker of nonpathogenic Th17 cells that have lost IL-17 secretion and functions as a negative regulator of Th17-induced inflammation in humans [44].
The toxicity testing did not reveal significant differences in hematology and biochemistry testing between the control group and the group receiving the $2 \mathrm{~F} 7$ anti-podoplanin neutralizing antibody. Moreover, although we expected to generate mouse IgG-specific immune response in monkey, no influence caused by administration of control mouse IgG1 was exhibited in hematological and blood analysis or histopathology examination. Therefore, it was suggested that the immune response against mouse IgG was not strongly induced as the toxicity was reflected. On the other hand, reproductive tissues from the monkeys treated with antibody, including testis, epididymis, and prostate, weighed more than those in control monkeys. Higher podoplanin expression was also detected in these reproductive tissues in monkeys (Figure 4C). We previously reported that podoplanin mRNA is highly expressed in the human testis and prostate [30]. Moreover, many podoplanin-expressing cells could be detected by $2 \mathrm{~F} 7$ in testis, epididymis and prostate (Figure 6B). Therefore, the relatively heavier reproductive tissues might have been caused by the podoplanin-neutralizing antibody. However, on histologic analysis, the difference in tissue weight was found to be due to greater sexual maturity in the antibodytreated monkeys rather than the inhibition of podoplanin (Figure 5). Cynomolgus monkeys vary considerably in the age at which sexual maturity is achieved, so either mature or immature testes can be observed in animals three to five years of age. Neither body weight nor age are predictive of the degree of testicular maturity that is seen histologically [45]. The accessory reproductive organs, i.e., the epididymis and prostate, develop once the testis reach sexual maturity [46]. Although we randomly assigned monkeys to either the control group or the antibodytreated group, we believe it was a coincidence that the $2 \mathrm{~F} 7$ treated group was more sexually mature than the control group. Regardless, our findings indicate that inhibition of podoplanin by the neutralizing antibody did not result in any acute toxicity in the monkeys.

In this study, we demonstrated that monkey podoplanin can induce platelet aggregation by binding CLEC-2, with the PLAG4 domain prominently involved as it is in humans. Inhibiting podoplanin with a neutralizing antibody targeting the PLAG4 domain exhibited no acute toxicity in cynomolgus monkeys. Our findings strongly suggest that podoplanin is a promising therapeutic target for treating cancer. A neutralizing antibody blocking podoplanin may be particularly useful in suppressing hematogenous metastasis.

\section{MATERIALS AND METHODS}

\section{Multiple sequence alignment}

Podoplanin and CLEC-2 sequences from various species were obtained from NCBI (https://www.ncbi. nlm.nih.gov/refseq/). These sequences were aligned, and 
homology was calculated from the p-distance by ClustalW multiple alignment in MEGA 4 software. A phylogenetic tree was constructed using the MEGA 4 program, and the genetic distance was inferred using the Neighbor-Joining method [47] with 1,000 bootstrap replicates.

\section{Plasmid construction}

Monkey podoplanin cDNA was obtained by reverse transcription polymerase chain reaction (RT-PCR) from JTC-12 cells (Riken BRC cell bank, Tsukuba, Japan). The PCR products were subcloned by Topo TA cloning (Invitrogen, Carlsbad, CA, USA) and, after sequence confirmation, cloned into pcDNA3 (Life Technologies, Carlsbad, CA, USA) or pGEX-6P-3 (GE Healthcare, Buckinghamshire, UK) plasmids using EcoRI restriction sites, resulting in pcDNA3 monkey podoplanin or pGEX6P-3 monkey podoplanin, respectively. The pcDNA3 construct was used to generate a stable transfectant (mkyPDPN) or mutated cDNAs with PLAG deletions

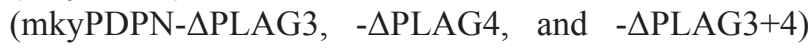
using a QuikChange site-directed mutagenesis kit (Agilent Technology, Santa Clara, CA, USA) according to the manufacturer's instructions. The pGEX-6P-3 monkey podoplanin construct was used for glutathione S-transferase (GST) -tagged point mutations of cDNA using epitope mapping as described previously [48]. A human podoplanin cDNA gene was cloned into the pcDNA3 vector and a stable transfectant (hPDPN) or human podoplanin mutants with PLAG deletions (hPDPN- $\triangle$ PLAG3, and - $\triangle$ PLAG4) were generated as previously described $[9,14]$. A plasmid encoding partial cDNA including the monkey podoplanin PLAG4 domain (XM_005544745.2, 409-510 b.p.; 157-170 aa; Integrated DNA Technologies, Iowa, USA) was repeatedly connected 21 times onto a pGEX-6P-3 vector and used as the immunogen to generate a monoclonal antibody (mAb) specific to the PLAG4 domain.

\section{Cell lines and culture conditions}

$\mathrm{CHO}$ cells were purchased from the American Type Culture Collection (ATCC, Manassas, VA, USA) and cultured in RPMI 1640 media (Wako, Osaka, Japan) containing 10\% FBS (Sigma-Aldrich, St. Louis, MO, USA). We established $\mathrm{CHO}$ cell lines stably transfected with vectors containing no podoplanin ( $\mathrm{CHO} / \mathrm{mock})$, wild-type human podoplanin (CHO/hPDPN-WT), wild-type monkey podoplanin (CHO/mkyPDPNWT) or the monkey podoplanin mutants with PLAG deletions (CHO/mkyPDPN- $\triangle$ PLAG3, - $\triangle$ PLAG4, and $-\Delta$ PLAG3+4) (Supplementary Figure 2A) by the same procedure as previously described [9]. The cells were cultured in medium containing $1 \mathrm{mg} / \mathrm{mL}$ of G418 (Life Technologies). JTC-12 cells (Riken BRC cell bank), derived from Macaca fascicularis kidneys, were cultured in DMEM low-glucose medium (Wako, Tokyo, Japan) containing 5\% FBS. Vero cells (ATCC), derived from Cercopithecus aethiops kidneys, were cultured in DMEM medium (Wako) containing $1 \mathrm{mM}$ sodium pyruvate and 10\% FBS. CV-1 and COS-7 cells (ATCC), derived from Cercopithecus aethiops kidneys, were cultured in MEM (Wako) containing x1 MEM nonessential amino acids (Wako), $1 \mathrm{mM}$ sodium pyruvate (Sigma), and 10\% FBS. Hybridoma cell lines secreting anti-podoplanin antibody were cultured in RPMI 1640 media (Wako) containing 10\% FBS (Sigma). DIG104.10H.1 hybridoma cells (JCRB cell bank, Osaka, Japan) were cultured in DMEM low-glucose medium (Wako) containing 10\% FBS.

\section{Animals}

Female BALB/c, BALB/c-nu/nu, and Jcl:ICR mice were purchased from Charles River (Kanagawa, Japan). All animal procedures were conducted in accordance with the guidelines of the Japanese Foundation for Cancer Research Animal Care and Use Committee. Male cynomolgus monkeys (Macaca fascicularis) were used for toxicity testing at the BoZo Research Center Inc. (Tokyo, Japan), conducted by the center's Institutional Animal Care and Use Committee.

\section{Flow cytometric analysis}

To analyze podoplanin expression, cells were harvested and treated with $1 \mu \mathrm{g} / \mathrm{mL}$ of anti-podoplanin antibodies (Sigma-Aldrich), followed by incubation with Alexa Flour 488-conjugated anti-mouse IgG $(\mathrm{H}+\mathrm{L})$ (Thermo Fisher Scientific, Waltham, MA, USA). For CLEC-2 binding analysis, cells were incubated with 0.4 to $1 \mu \mathrm{g} / \mathrm{mL}$ of (His) ${ }_{10}$-tagged human CLEC-2 or $5 \mu \mathrm{g}$ / $\mathrm{mL}$ of (His) ${ }_{10}$-tagged mouse CLEC-2 (R \& D Systems, Minneapolis, MN, USA), followed by incubation with Alexa Flour 488-conjugated anti-penta-His antibody (Qiagen, Venlo, Netherlands). For the antibody inhibition assay, cells were incubated with $100 \mu \mathrm{g} / \mathrm{mL}$ of anti-podoplanin $\mathrm{mAbs}$ or control IgG for $30 \mathrm{~min}$ on ice before incubation with human or mouse CLEC-2. Fluorescence intensity was measured using a Cytomics FC500 flow cytometry system (Beckman Coulter, CA, USA) and analyzed with FlowJo software (Treestar, Inc., San Carlos, CA).

\section{Platelet aggregation assay}

Murine whole blood from cardiac puncture in Jcl:ICR mice killed with sevoflurane was mixed with heparin or $3.2 \%(\mathrm{w} / \mathrm{v})$ sodium citrate solution to produce platelet-rich plasma. The platelet aggregation rate was estimated by a previously described procedure [14].

\section{Western blot analysis}

Cells were lysed in lysis buffer $(0.1 \mathrm{M}$ Tris- $\mathrm{HCl}$ pH 7.5, 10\% glycerol, and 1\% sodium dodecyl sulfate 
[SDS]) and boiled for $5 \mathrm{~min}$. Total protein concentrations were determined with BCA Protein Assay Reagent (Pierce, Rockford, IL, USA). Each cell lysate or GSTtagged mkyPDPN-WT and its point mutants extracted from BL21 (DE3) E. coli (Thermo Fisher Scientific) were treated with SDS sample buffer, electrophoresed on an SDS-polyacrylamide gel (Nacalai Tesque), and transferred onto PVDF membranes (EMD Millipore, MA, USA). The membranes were incubated with primary antibodies to podoplanin (D2-40 [M3619, Dako, CA, USA], MS-1, PG4D2, 1F6, 2F7, and 3F4), $\beta$-actin (clone, AC-15, Sigma-Aldrich), or GST (clone 4C10, ab73934, Abcam, Cambridge, UK), followed by treatment with horseradish peroxidase-conjugated anti-mouse IgG (RPN2232, GE Healthcare), and finally reacted with ECL Prime Western Blotting Detection reagent (GE Healthcare). The proteins were visualized with enhanced chemiluminescence by an Amersham Imager 600 (GE Healthcare).

\section{Production and purification of anti-PLAG4 monoclonal antibodies}

A monkey podoplanin cDNA region encoding amino acids 76 to 89 (226-267 bp) was tandemly connected 21 times in a pGEX-6P-3 vector (GE Healthcare), replicated in BL21 (DE3) E. coli as the GST-tagged monkey podoplanin peptide (76-89 aa), and purified using glutathione sepharose (GE Healthcare). Sixweek-old female BALB/c mice were immunized by neck and ventral subcutaneous injections of the GST-tagged peptide with Titer MAX Gold adjuvant (Titer MAX, Norcross, GA, USA). Cell fusion was performed by the procedure described previously [14]. Positive clones were subcloned three times by limiting dilution, and some were purified from ascites as described previously [48]. For the acute toxicity test with cynomolgus monkeys, large-scale purification of control IgG1 and 2F7 antibody was conducted. Six-week-old female $\mathrm{BALB} / \mathrm{c}-n u / n u$ mice were injected intraperitoneally with 2F7-secreting hybridomas or control IgG1-secreting hybridomas (DIG104.10H.1) suspended in Hanks' Balanced Salt Solution (HBSS, Gibco). Ascitic fluid was collected for purification of the antibody by salting out with ammonium sulfate and performing affinity column chromatography with protein G using AKTA Explorer 10S (GE Healthcare). The IgG isotype was determined with a Mouse Monoclonal Antibody Isotyping Test Kit (AbD Serotec, Oxford, UK).

\section{Inhibitory effect of anti-podoplanin antibodies on pulmonary metastasis}

Six-week-old female BALB/c-nu/nu mice were intravenously injected with anti-podoplanin mAbs (10 $\mu \mathrm{g} /$ mouse) through the lateral tail vein the day before cell injection. $\mathrm{CHO} / \mathrm{mock}$ or $\mathrm{CHO} / \mathrm{mkyPDPN}-\mathrm{WT}$ cells were suspended in HBSS (Gibco) and injected intravenously $\left(2.5 \times 10^{5}\right.$ cells/mouse $)$ through the lateral tail vein. The mice were killed 16 days later. The lungs were removed and stained with saturated picric acid solution. The numbers of metastatic foci on the lung surface were counted.

\section{Quantitative reverse transcription polymerase chain reaction}

Quantitative reverse transcription polymerase chain reaction (qRT-PCR) was performed using a FastStart Essential DNA Green Master (Roche, Basel, Switzerland). A Monkey Tissue cDNA Panel (Zyagen) was used to screen for monkey $p d p n$ and $\beta$-actin expression. Standard curves were generated from a dilution series of cDNA from lymph nodes. Primer pairs used in qRT-PCR were as follows: monkey $p d p n$ forward, 5'-AAATGTCAGGAAGGTACTCG-3' and reverse, 5'-GCCGGGCAAGTGTTCCAC-3'; monkey $\beta$-actin forward, 5'-CCAACCGCGAGAAGATGA-3' and reverse, 5'-CCAGAGGCGTACAGGGACAG-3'.

\section{Toxicity test}

Male cynomolgus monkeys (Macaca fascicularis) (each group has $\mathrm{N}=2$ ), which were 41 to 44 months of age and 3.64 to $4.22 \mathrm{~kg}$ of body weight, were intravenously injected with $100 \mathrm{mg} / \mathrm{kg}$ of $2 \mathrm{~F} 7$ antibodies or control IgG1 through the cephalic vein. Blood was collected 7 days before and 6 and 13 days after the injection for hematology and biochemistry analysis. The monkeys were euthanized 2 weeks after injection and tissues and organs were weighed. Tissue sections were fixed in $10 \%$ neutral buffer formalin and stained with hematoxylineosin. Analysis of tissue specimens was performed by two independent pathologists (Drs. Kanda and Furuta) who were blind to diagnosis.

\section{Immunohistochemical staining of anti- podoplanin antibodies}

To estimate the reactivity of $2 \mathrm{~F} 7$ to each monkey tissues, we compared the reactivity of $2 \mathrm{~F} 7$ with the commercially available antibody to human podoplanin. Immunohistochemical staining was performed on paraffin sections using a polymer peroxidase method. Briefly, deparaffinized and rehydrated sections were activated antigenicity of antigen in EnVision FLEX TARGET RETRIEVAL SOLUTION High pH (Dako) for $45 \mathrm{~min}$ at $95^{\circ} \mathrm{C}$ and cooled for $30 \mathrm{~min}$. Sections were treated with 3\% hydrogen peroxide in methanol for $5 \mathrm{~min}$ to block endogenous peroxidase activity. After rinsing in PBS, sections were incubated with primary antibodies to podoplanin (2F7, D2-40 [Dako], FL-162 [sc-134482, 
Santa Cruz Biotechnology, CA, USA], NZ-1 [012-25863, Wako], LpMab-12 [MABT841, EMD Millipore] or control IgG) overnight. Thereafter, they were incubated with the Histofine Simple Stain MAX PO (MULTI) kit (Nichirei, Tokyo, Japan) for 30min. Sections were stained with 3,3-diaminobenzidine tetrahydrochloride and counterstained with hematoxylin.

\section{Statistical analysis}

The Mann-Whitney $U$-test was performed to determine the statistical significance of comparisons. Statistical significance was assumed for ${ }^{*} P<0.05$ or ${ }^{* *} P$ $<0.01$. All statistical tests were two-sided.

\section{ACKNOWLEDGMENTS}

We thank Dr. Hiroaki Kanda, Dr. Reiko Furuta, and Ms. Satoko Baba for histological examinations of monkey specimens.

\section{CONFLICTS OF INTEREST}

NF is receiving a research grant from Api, Co., Ltd, who are developing anti-podoplanin antibodies as a therapeutic agent. Other authors declare that there is no conflicts of interest that could be perceived as prejudicing the impartiality of the research reported.

\section{FUNDING}

Authors were supported by the Medical Research and Development Programs Focused on Technology Transfer, Acceleration Transformative Research for Medical Innovation (ACT-MS, No. 17im021060h0002; to NF) and Project for Cancer Research and Therapeutic Evolution (P-CREATE, No. $17 \mathrm{~cm} 0106205 \mathrm{~h} 0002$; to NF) from the Japan Agency for Medical Research and Development (AMED) and were supported in part by MEXT KAKENHI Grant Number JP17H06327 (Grant-inAid for Scientific Research on Innovative Areas, to NF) and JSPS KAKENHI Grant Number JP16H06276.

\section{REFERENCES}

1. Fidler IJ. Metastasis: quantitative analysis of distribution and fate of tumor emboli labeled with 125 I-5-iodo-2'deoxyuridine. J Natl Cancer Inst. 1970; 45:773-782.

2. Gay LJ, Felding-Habermann B. Contribution of platelets to tumour metastasis. Nat Rev Cancer. 2011; 11:123-134.

3. Gasic GJ, Gasic TB, Stewart CC. Antimetastatic effects associated with platelet reduction. Proc Natl Acad Sci U S A. 1968; 61:46-52.

4. Kunita A, Kashima TG, Morishita Y, Fukayama M, Kato Y, Tsuruo T, Fujita N. The platelet aggregation-inducing factor aggrus/podoplanin promotes pulmonary metastasis. Am J Pathol. 2007; 170:1337-1347.

5. Klerk CP, Smorenburg SM, Otten HM, Lensing AW, Prins MH, Piovella F, Prandoni P, Bos MM, Richel DJ, van Tienhoven G, Buller HR. The effect of low molecular weight heparin on survival in patients with advanced malignancy. J Clin Oncol. 2005; 23:2130-2135.

6. Lazo-Langner A, Goss GD, Spaans JN, Rodger MA. The effect of low-molecular-weight heparin on cancer survival. A systematic review and meta-analysis of randomized trials. J Thromb Haemost. 2007; 5:729-737.

7. Ma T, Yang B, Matthay MA, Verkman AS. Evidence against a role of mouse, rat, and two cloned human t1alpha isoforms as a water channel or a regulator of aquaporin-type water channels. Am J Respir Cell Mol Biol. 1998; 19:143-149.

8. Zimmer G, Oeffner F, Von Messling V, Tschernig T, Gröness HJ, Klenk HD, Herrler G. Cloning and characterization of gp36, a human mucin-type glycoprotein preferentially expressed in vascular endothelium. Biochem J. 1999; 341:277-284.

9. Kato Y, Fujita N, Kunita A, Sato S, Kaneko M, Osawa M, Tsuruo T. Molecular identification of Aggrus/T1alpha as a platelet aggregation-inducing factor expressed in colorectal tumors. J Biol Chem. 2003; 278:51599-515605.

10. Suzuki-Inoue K, Kato Y, Inoue O, Kaneko MK, Mishima K, Yatomi Y, Yamazaki Y, Narimatsu H, Ozaki Y. Involvement of the snake toxin receptor CLEC-2, in podoplanin mediated platelet activation, by cancer cells. J Biol Chem. 2007; 282:25993-26001.

11. Takagi S, Sato S, Oh-hara T, Takami M, Koike S, Mishima Y, Hatake K, Fujita N. Platelets promote tumor growth and metastasis via direct interaction between Aggrus/podoplanin and CLEC-2. PLoS One. 2013; 8:e73609.

12. Suzuki-Inoue K, Fuller GL, Garcia A, Eble JA, Pohlmann S, Inoue O, Gartner TK, Hughan SC, Pearce AC, Laing GD, Theakston RD, Schweighoffer E, Zitzmann N, et al. A novel Syk-dependent mechanism of platelet activation by the C-type lectin receptor CLEC-2. Blood. 2006; 107:542-549.

13. Suzuki-Inoue K. Essential in vivo roles of the platelet activation receptor CLEC-2 in tumour metastasis, lymphangiogenesis and thrombus formation. J Biochem. 2011; 150:127-132.

14. Sekiguchi T, Takemoto A, Takagi S, Takatori K, Sato S, Takami M, Fujita N. Targeting a novel domain in podoplanin for inhibiting platelet-mediated tumor metastasis. Oncotarget. 2016; 7:3934-3946. https://doi. org/10.18632/oncotarget.6598.

15. Nagae M, Morita-Matsumoto K, Kato M, Kaneko MK, Kato Y, Yamaguchi Y. A platform of C-type lectin-like receptor CLEC-2 for binding O-glycosylated podoplanin and nonglycosylated rhodocytin. Structure. 2014; 22:1711-1721.

16. Shirai T, Inoue O, Tamura S, Tsukiji N, Sasaki T, Endo H, Satoh K, Osada M, Sato-Uchida H, Fujii H, Ozaki Y, 
Suzuki-Inoue K. C-type lectin-like receptor 2 promotes hematogenous tumor metastasis and prothrombotic state in tumor-bearing mice. J Thromb Haemost. 2017; 15:513-525.

17. Chang YW, Hsieh PW, Chang YT, Lu MH, Huang TF, Chong KY, Liao HR, Cheng JC, Tseng CP. Identification of a novel platelet antagonist that binds to CLEC-2 and suppresses podoplanin-induced platelet aggregation and cancer metastasis. Oncotarget. 2015;6:42733-42748. https://doi.org/10.18632/oncotarget.5811.

18. Miyata K, Takemoto A, Okumura S, Nishio M, Fujita N. Podoplanin enhances lung cancer cell growth in vivo by inducing platelet aggregation. Sci Rep. 2017; 7:4059.

19. Takemoto A, Miyata K, Fujita N. Platelet-activating factor podoplanin: from discovery to drug development. Cancer Metastasis Rev. 2017; 36:225-234.

20. Suzuki-Inoue K, Inoue O, Ding G, Nishimura S, Hokamura K, Eto K, Kashiwagi H, Tomiyama Y, Yatomi Y, Umemura $\mathrm{K}$, Shin Y, Hirashima M, Ozaki Y. Essential in vivo roles of the C-type lectin receptor CLEC-2: embryonic/neonatal lethality of CLEC-2-deficient mice by blood/ lymphatic misconnections and impaired thrombus formation of CLEC-2 deficient platelets. J Biol Chem. 2010; 285:24494-24507.

21. Ramirez MI, Millien G, Hinds A, Cao Y, Seldin DC, Williams MC. T1alpha, a lung type I cell differentiation gene, is required for normal lung cell proliferation and alveolus formation at birth. Dev Biol. 2003; 256:61-72.

22. Schacht V, Ramirez MI, Hong YK, Hirakawa S, Feng D, Harvey N, Williams M, Dvorak AM, Dvorak HF, Oliver G, Detmar M. T1alpha/podoplanin deficiency disrupts normal lymphatic vasculature formation and causes lymphedema. EMBO J. 2003; 22:3546-3556.

23. Staines KA, Javaheri B, Hohenstein P, Fleming R, Ikpegbu E, Unger E, Hopkinson M, Buttle DJ, Pitsillides AA, Farquharson C. Hypomorphic conditional deletion of E11/ Podoplanin reveals a role in osteocyte dendrite elongation. J Cell Physiol. 2017; 232:3006-3019.

24. Chellman GJ, Bussiere JL, Makori N, Martin PL, Ooshima Y, Weinbauer GF. Developmental and reproductive toxicology studies in nonhuman primates. Birth Defects Res B Dev Reprod Toxicol. 2009; 86:446-462.

25. Perelman P, Johnson WE, Roos C, Seuánez HN, Horvath JE, Moreira MA, Kessing B, Pontius J, Roelke M, Rumpler Y, Schneider MP, Silva A, O'Brien SJ, Pecon-Slattery J. A molecular phylogeny of living primates. PLoS Genet. 2011; 7:e1001342.

26. Ogasawara S, Kaneko MK, Price JE, Kato Y. Characterization of anti-podoplanin monoclonal antibodies: critical epitopes for neutralizing the interaction between podoplanin and CLEC-2. Hybridoma (Larchmt). 2008; 27:259-267.

27. Martin-Villar E, Scholl FG, Gamallo C, Yurrita MM, Munoz-Guerra M, Cruces J, Quintanilla M. Characterization of human PA2.26 antigen (T1alpha-2, podoplanin), a small membrane mucin induced in oral squamous cell carcinomas. Int J Cancer. 2005; 113:899-910.

28. Kimura N, Kimura I. Podoplanin as a marker for mesothelioma. Pathol Int. 2005; 55:83-86.

29. Mishima K, Kato Y, Kaneko MK, Nishikawa R, Hirose $\mathrm{T}$, Matsutani M. Increased expression of podoplanin in malignant astrocytic tumors as a novel molecular marker of malignant progression. Acta Neuropathol. 2006; 111: 483-488.

30. Takagi S, Oh-hara T, Sato S, Gong B, Takami M, Fujita N. Expression of Aggrus/podoplanin in bladder cancer and its role in pulmonary metastasis. Int J Cancer. 2014; 134: 2605-2614.

31. Breiteneder-Geleff S, Soleiman A, Kowalski H, Horvat R, Amann G, Kriehuber E, Diem K, Weninger W, Tschachler E, Alitalo K, Kerjaschki D. Angiosarcomas express mixed endothelial phenotypes of blood and lymphatic capillaries: podoplanin as a specific marker for lymphatic endothelium. Am J Pathol. 1999; 154:385-394.

32. Shibahara J, Kashima T, Kikuchi Y, Kunita A, Fukayama M. Podoplanin is expressed in subsets of tumors of the central nervous system. Virchows Arch. 2006; 448:493-499.

33. Kato Y, Sasagawa I, Kaneko M, Osawa M, Fujita N, Tsuruo T. Aggrus: a diagnostic marker that distinguishes seminoma from embryonal carcinoma in testicular germ cell tumors. Oncogene. 2004; 23:8552-8556.

34. Kunita A, Kashima TG, Ohazama A, Grigoriadis AE, Fukayama M. Podoplanin is regulated by AP-1 and promotes platelet aggregation and cell migration in osteosarcoma. Am J Pathol. 2011; 179:1041-1049.

35. Yuan P, Temam S, El-Naggar A, Zhou X, Liu DD, Lee JJ, Mao L. Overexpression of podoplanin in oral cancer and its association with poor clinical outcome. Cancer. 2006; 107:563-569.

36. Xu Y, Ogose A, Kawashima H, Hotta T, Ariizumi T, Li G, Umezu H, Endo N. High-level expression of podoplanin in benign and malignant soft tissue tumors: Immunohistochemical and quantitative real-time RT-PCR analysis. Oncol Rep. 2011; 25:599-607.

37. Pula B, Jethon A, Piotrowska A, Gomulkiewicz A, Owczarek T, Calik J, Wojnar A, Witkiewicz W, Rys J, Ugorski M, Dziegiel P, Podhorska-Okolow M. Podoplanin expression by cancer-associated fibroblasts predicts poor outcome in invasive ductal breast carcinoma. Histopathology. 2011; 59:1249-1260.

38. Astarita JL, Acton SE, Turley SJ. Podoplanin: emerging functions in development, the immune system, and cancer. Front Immunol. 2012; 3:283.

39. Yurugi Y, Wakahara M, Matsuoka Y, Sakabe T, Kubouchi Y, Haruki T, Nosaka K, Miwa K, Araki K, Taniguchi Y, Shiomi T, Nakamura H, Umekita Y. Podoplanin expression in cancer-associated fibroblasts predicts poor prognosis in patients with squamous cell carcinoma of the lung. Anticancer Res. 2017; 37:207-213. 
40. Takemoto A, Okitaka M, Takagi S, Takami M, Sato S, Nishio M, Okumura S, Fujita N. A critical role of platelet TGF- $\beta$ release in podoplanin-mediated tumour invasion and metastasis. Sci Rep. 2017; 7:42186.

41. Breiteneder-Geleff S, Matsui K, Soleiman A, Meraner P, Poczewski H, Kalt R, Schaffner G, Kerjaschki D. Podoplanin, novel 43-kd membrane protein of glomerular epithelial cells, is down-regulated in puromycin nephrosis. Am J Pathol. 1997; 151:1141-1152.

42. Matsui K, Breiteneder-Geleff S, Kerjaschki D. Epitopespecific antibodies to the $43-\mathrm{kD}$ glomerular membrane protein podoplanin cause proteinuria and rapid flattening of podocytes. J Am Soc Nephrol. 1998; 9:2013-2026.

43. Bertozzi CC, Schmaier AA, Mericko P, Hess PR, Zou Z, Chen M, Chen CY, Xu B, Lu MM, Zhou D, Sebzda E, Santore MT, Merianos DJ, et al. Platelets regulate lymphatic vascular development through CLEC-2-SLP-76 signaling. Blood. 2010; 116:661-670.
44. Nylander AN, Ponath GD, Axisa PP, Mubarak M, Tomayko M, Kuchroo VK, Pitt D, Hafler DA. Podoplanin is a negative regulator of Th17 inflammation. JCI Insight. 2017; 2.

45. Haruyama E, Suda M, Ayukawa Y, Kamura K, Mizutamari M, Ooshima Y, Tanimoto A. Testicular development in cynomolgus monkeys. Toxicol Pathol. 2012; 40:935-942.

46. Haruyama E, Ayukawa Y, Kamura K, Mizutamari M, Ooshima Y, Tanimoto A. Morphometric examination for development of reproductive organs in male cynomolgus monkeys. Toxicol Pathol. 2012; 40:918-925.

47. Saitou N, Nei M. The neighbor-joining method: a new method for reconstructing phylogenetic trees. Mol Biol Evol. 1987; 4:406-425.

48. Nakazawa Y, Takagi S, Sato S, Oh-hara T, Koike S, Takami M, Arai H, Fujita N. Prevention of hematogenous metastasis by neutralizing mice and its chimeric anti-Aggrus/ podoplanin antibodies. Cancer Sci. 2011; 102:2051-2057. 